\title{
Rechtsetzung und Kontrolle der Gesetzesdurchführung in der V.R. China
}

\author{
Von Barbara Darimont, München
}

\begin{abstract}
"Therefore it is said:
Law must remain constant.

It is the determining factor

as to whether one survives or perishes,

has order or chaos."

Aus dem Buch "Guanzi”, Kapitel 45
\end{abstract}

Mit den Wirtschaftsreformen seit Ende der 70er Jahre wurde in der V.R. China aktiv Recht gesetzt. In der Zeit von 1979 bis 2001 wurden über 370 Gesetze und entsprechende Beschlüsse, über 800 Verwaltungsrechtsnormen und über 7.000 lokale Rechtsnormen erlassen. ${ }^{2} \mathrm{Zu}$ Beginn der Wirtschaftsreformen war es gerechtfertigt, den Focus auf die Quantität der Rechtsnormen zu legen, um über "Recht, das als Grundlage dient, zu verfügen"3 (youfa yiju). Dieser pragmatische Ansatz wird in den letzten Jahren jedoch in Frage gestellt, da es in der V.R. China zu einer enormen Rechtsdiversifikation gekommen ist. Besonders problematisch erweist sich die mangelnde Regelung des Rechtsetzungsverfahren und der Normenkontrolle. Die Normenhierarchie und rechtliche Terminologie der V.R. China sind ebenfalls konfus, weil nicht nur die Bezeichnungen uneinheitlich sind, sondern die Rechtsnormen aus verschiedenen Quellen stammen und unterschiedliche Wirkung haben.

"Guanzi” gehört zu den frühen Werken der Legalisten. Ein Politiker des Staates Qi, Guan Zhong (gest. 645 v. Chr.) soll der Autor sein. Die gegenwärtige Fassung wird Liu Xiang zugerechnet, der es ungefähr 26. v. Chr. kompilierte. Übersetzung von Rickett, W. Allyn, Guanzi. Political, Economic, and Philosophical Essays from Early China. A study and translation, New Jersey, 1998, S. 146.

RMRB vom 3.12.2001, 7; vgl. Li, Yuwen/Otto, Jan-Michiel, Central and Local Law-Making: Studying China's Experience in: Vermeer, Eduard B./d'Hooghe, Ingrid, China's Legal Reforms and Their Political Limits, London, 2002, S. 25.

Zhu, Weijiu, Rechtsaufsicht sowie politisch demokratische Aufsicht in China (Zhongguo de falü jiandu yu zhengzhi minzhu jiandu), in: Forum für Politik und Recht (Zhengfa luntan) 2000, Beijing, Heft 1, S. 145 ff. 
Um diesen Missstand zu beseitigen, wurde am 15.3.2000 vom Nationalen Volkskongress (im Folgenden NVK) das "Gesetz der V.R. China über die Gesetzgebung"4 verabschiedet. Es trat am 1.7.2000 in Kraft. Geregelt werden Erlass, Änderung und Aufhebung von Gesetzen und lokalen Vorschriften. Diese sind zwar in Art. 62 ff. der "Verfassung der V.R. China"5 aus dem Jahr 1982 festgelegt, sie werden aber durch das Gesetzgebungsgesetz konkretisiert. Im Gesetzgebungsgesetz werden die Verhältnisse der einzelnen Rechtsvorschriften untereinander, das Gesetzgebungsverfahren und die Auslegung von Gesetzen festgelegt. Zur weiteren Konkretisierung wurden am 16.12.2001 die "Regeln über das Festlegungsverfahren von Verwaltungsrechtsnormen"6 und die "Regeln über das Festlegungsverfahren von Vorschriften"7 verabschiedet. Mit diesen Regeln wird das Bemühen ersichtlich, eine stärkere Rechtsvereinheitlichung durchzuführen.

\section{Rechtsetzungsverfahren}

Der NVK ist nach Art. 57 f. der Verfassung das "höchste Organ der Staatsmacht". Zusammen mit seinem Ständigen Ausschuss übt der NVK die legislative Gewalt des Staates aus. Darüber hinaus gehören zu den Aufgaben des NVK und seines Ständigen Ausschusses Gesetzesauslegungen, Entscheidungen bei wichtigen Angelegenheiten, Ernennungen von Personal und die Normenkontrolle. ${ }^{8}$ Die bis zu 3.000 Abgeordneten des NVK werden alle fünf Jahre von indirekt gewählten Vertretern der Provinzkongresse bestimmt. ${ }^{9}$ Von den derzeit 2.985 Mitgliedern des NVK sind 72,99\% in der KP. ${ }^{10}$ Das durchschnittliche Alter der Abgeordneten beträgt 52,27 Jahre. Der Frauenanteil liegt bei 21,8 \%. ${ }^{11}$ Aufgrund seiner Größe findet der NVK nur einmal im Jahr statt. Das Arbeitsorgan des NVK ist sein Ständiger Ausschuss, der zur Zeit mit ungefähr 150 Mitgliedern alle zwei Monate tagt.

Zhongguo Renmin Gongheguo lifa fa, FZRB (Legal Daily, chinesischsprachige Tageszeitung, Beijing) vom 19.3.2000; dt. Übersetzung in: China aktuell 2000, Hamburg, S. 939 ff. von Heuser, Robert.

Zhongguo Renmin Gongheguo xianfa, mit den entsprechenden Verfassungsänderungen in: RMRB (Tageszeitung des Volkes, chinesischsprachige Tageszeitung, Beijing) vom 17.3.1999; engl. Übersetzung in: Laws of the People's Republic of China 1999, Beijing, S. 3 ff.

6 Xingzheng fagui zhiding chengxu tiaoli - Guowuyuanling Nr. 321, GGB (Guowuyuan Gongbao, Amtsblatt des Staatsrats der V.R. China, Beijing) 2002, Heft 1, S. 5 ff.

Guizhang zhiding chengxu tiaoli - Guowuyuanling Nr. 322, GGB 2002, Heft 1, S. 7 ff.

8 Art. 62 ff. der Verfassung; Cai, Dingjian, Das System der Nationalen Volkskongresse (Zhongguo Renmin Daibiao Dahu zhidu), Rechtsverlag, Beijing, 1998, S. 247 ff.

Ausführlich zum NVK s. Tanner, Murray Scott, The Politics of Lawmaking in Post-Mao China, Oxford, 1999, S. 72 ff.

10

$\mathrm{CD}(\mathrm{CD}=$ China Daily, englischsprachige Tageszeitung, Beijing) vom 28.2.2003.

11

Cai, Dingjian (Fn. 8), S. 227 f. 
Das Gesetzgebungsgesetz weist den Rechtsnormen eine klare Rangordnung zu. ${ }^{12}$ Die Prinzipien des Gesetzesvorranges und des Gesetzesvorbehaltes, die auch in der Verfassung geregelt sind, wurden mit dem Gesetzgebungsgesetz gestärkt. Gesetze gehen administrativen Rechtsnormen und diese lokalen Vorschriften vor. Ferner gilt der Vorrang der spezielleren vor der allgemeineren Norm. Auch der Grundsatz, dass alte Vorschriften vor neuen gleichrangigen weichen, ist festgelegt. Bei speziellen Bestimmungen zum Schutz der Bürger, juristischer Personen und weiterer Organisationen gilt generell das Verbot der Rückwirkung von Gesetzen und Vorschriften.

\subsection{Gesetzgebungsverfahren bei formellen Gesetzen}

Das Initiativrecht für Gesetzesvorschläge haben der NVK, sein Ständiger Ausschuss, die verschiedenen Fachausschüsse des NVK, der Staatsrat, die Zentrale Militärkommission, das Oberste Volksgericht und die Oberste Volksstaatsanwaltschaft. ${ }^{13}$ Außerdem kann eine Gruppe von mehr als 30 Delegierten des NVK einen Gesetzentwurf dem NVK vorschlagen. Das Präsidium des NVK entscheidet über die Annahme des Vorschlages. ${ }^{14}$ Beim Ständigen Ausschuss des NVK reicht es, wenn mehr als 10 Mitglieder einen Gesetzesvorschlag unterbreiten; in diesem Fall urteilt die Vorsitzendenkonferenz des Ständigen Ausschusses über die Gesetzesinitiative. ${ }^{15}$

In der Regel werden Gesetze vom Staatsrat initiiert. ${ }^{16}$ Anschließend wird meistens eine Kommission zur Erarbeitung eines Gesetzentwurfs eingerichtet oder der Entwurf wird direkt von der Arbeitskommission für Gesetzgebung des Ständigen Ausschusses des NVK (fagongwei) oder den entsprechenden Abteilungen des Staatsrats formuliert. Im Laufe des Ausarbeitungsprozesses kooperieren die Arbeitskommission für Gesetzgebung des Ständigen Ausschusses des NVK und die entsprechenden Abteilungen des Staatsrats eng miteinander. $^{17}$

Meistens sind Verhandlungen der betroffenen Ministerien und staatlichen Abteilungen einer Gesetzesvorlage vor dem NVK und der Verabschiedung von Gesetzen vorgeschaltet. Ein rechtlich festgelegtes Verfahren existiert im Bezug auf diesen Beratungsvorgang

$\begin{array}{ll}12 & \text { Art. } 78 \text { ff. des Gesetzgebungsgesetzes. } \\ 13 & \text { Art. } 12 \text { des Gesetzgebungsgesetzes. } \\ 14 & \text { Art. } 13 \text { des Gesetzgebungsgesetzes. } \\ 15 & \text { Art. } 25 \text { des Gesetzgebungsgesetzes. } \\ 16 & \text { Tanner, Murray Scott (Fn. 9), S. 130. } \\ 17 & \text { Li, Yuwen/Otto, Jan-Michiel (Fn. 2), S. } 11 \text { f. }\end{array}$ 
nicht. ${ }^{18}$ Für Gesetzesentwürfe, über die der Ständige Ausschuss des NVK entscheidet, sind drei Lesungen vorgesehen. ${ }^{19}$ Im Verhandlungsprozess ist es möglich, dass Gesetzentwürfe aufgrund von divergierenden Interessen nicht zur Abstimmung kommen und für lange Zeit dem NVK nicht wieder vorgelegt werden. Ein Beispiel ist das Arbeitsgesetz, das schon 1984/85 als Entwurf den Abteilungen des NVK vorgelegt wurde. Die entsprechende Abteilung verlangte eine Überarbeitung, da der Entwurf nicht die für notwendig gehaltenen Reformen enthielt, und leitete ihn zurück an das Ministerium für Arbeit und soziale Sicherheit. Das Arbeitsgesetz wurde erst zehn Jahre später, nämlich im Jahr 1994, verabschiedet. $^{20}$

Der Nationale Volkskongress (NVK) verabschiedet Verfassungsänderungen und grundlegende Gesetze (jiben falü). ${ }^{21}$ Diese grundlegende Gesetze des Straf-, Zivil- und Öffentlichen Rechts dürfen nur vom NVK erlassen werden. Eine Legaldefinition für grundlegende Gesetze existiert nicht. ${ }^{22}$ Sie sollen gesellschaftliche Beziehungen, die für den Staat und das gesellschaftliche Leben eine umfassende, langfristige, allgemeine und fundamentale Bedeutung haben, ${ }^{23}$ regeln und normieren. Ein Beispiel für ein grundlegendes Gesetz ist das Gesetzgebungsgesetz.

Dem Ständigen Ausschuss des NVK obliegt es, die anderen formalen Gesetze zu erlassen. Außerdem legt er die Verfassung und Gesetze aus. ${ }^{24}$

Im Gesetzgebungsgesetz wurde in Art. 8 ein Gesetzesvorbehalt (falï baoliu) ${ }^{25}$ eingeführt, der sich auf förmliche Gesetze bezieht. Dieser Vorbehalt erstreckt sich auf folgende Gegenstände:

Allerdings ist in $\S 34$ der "Regeln über das Festlegungsverfahren für Verwaltungsrechtsnormen" festgelegt, dass bei Gesetzesentwürfen, die der Staatsrat ausarbeitet, um sie dem NVK oder seinem Ständigen Ausschuss zur Diskussion und Beschlussfassung vorzulegen, das Verfahren entsprechend den o.g. Regeln stattzufinden hat.

19

20

21

22 
1. Staatssouveränität;

2. Aufbau und Organisation der Volkskongresse, -regierungen, -gerichte sowie der Volksstaatsanwaltschaft;

3. Organisation der Selbstverwaltung von ethnischen Gebietsautonomien, von Sonderverwaltungszonen und der kommunalen Selbstverwaltung;

4. Verbrechen und deren Bestrafung;

5. Entzug der politischen Rechte von Bürgern;

6. Zwangsmaßnahmen und Strafen;

7. Einzug von nicht-staatlichem Vermögen;

8. Grundzüge des Zivil-, Wirtschaft-, Finanz- und Steuerrechts;

9. Prozess- und Schiedsverfahren;

10.Weitere, vom NVK oder seinem ständigen Ausschuss festgelegte Bereiche.

In der juristischen Literatur Chinas wird über eine weitere Differenzierung des Gesetzesvorbehaltes diskutiert. Danach fallen die Gegenstände unter Punkt 1. bis 3. und 7. unter einen "totalen Vorbehalt" (juedui baoliu) des Gesetzes, während es sich bei den anderen Gegenständen um einen "relativen Vorbehalt" (xiangdui baoliu) handelt. Bei einem relativen Vorbehalt können Teilbereiche aufgrund einer Ermächtigung geregelt werden. ${ }^{26}$ Eine weitere Differenzierung des Gesetzesvorbehaltes wird in Zukunft wahrscheinlich folgen. Es wäre beispielsweise sinnvoll, einige Gegenstände durch den Vorbehalt eines grundlegenden Gesetzes oder der Verfassung zu schützen.

Ein Katalog über die Kompetenztitel wurde jedoch weder in der Verfassung noch im Gesetzgebungsgesetz aufgenommen. Es stellt sich daher immer noch die Frage, welche Materien durch förmliches Gesetz und welche durch andere Rechtsnormen zu regeln sind. ${ }^{27}$

Verfassungsänderungen müssen mit einer Zweidrittelmehrheit aller Abgeordneten des NVK und Gesetze mit einer einfachen Mehrheit gemäß Art. 64 der Verfassung angenommen werden. Gesetze, deren Verabschiedung fraglich ist, werden nicht auf die Tagesordnung gesetzt, so dass garantiert ist, dass alle zur Verabschiedung vorgelegten Gesetze angenommen werden. Deshalb wird dieses Verfahren durch den NVK und seinen Ständigen Ausschuss von ausländischen Wissenschaftlern als "Gummistempel-Maschinerie"28 bezeichnet, da die vom Politbüro genehmigten Gesetzesentwürfe widerspruchslos ange-

26

27

28

Ying, Songnian (Fn. 25), S. 6.

Vgl. Heuser, Robert, An den Grenzen des Wandels: Zum chinesischen Gesetzgebungsgesetz, in: Hofmann, Mahulena/Küpper, Herbert (Hrsg.), Kontinuität und Neubeginn. Staat und Recht in Europa zu Beginn des 21. Jahrhunderts. Festschrift für Georg Brunner aus Anlass seines 65. Geburtstags, Baden-Baden, 2001, S. 62 ff., 62.

Tanner, Murray Scott (Fn. 9), S. 1; vgl. Holbig, Heike, Loyale Ambitionen, Die Jahrestagung des Nationalen Volkskongresses, China aktuell, März 2001, S. 279 ff., 283. 
nommen würden. Es mehreren sich aber Anzeichen für eine gegenläufige Entwicklung, denn der NVK lehnte die Verabschiedung zweier Gesetze in jüngster Zeit ab. ${ }^{29}$

\subsection{Verfahren bei Rechtsnormen der Exekutive}

Als höchstes Organ der Staatsverwaltung ${ }^{30}$ ist der Staatsrat zuständig für den Erlass von Verwaltungsrechtsnormen, die zur Durchführung von Gesetzen erforderlich sind. Auch die Verabschiedung von ausführenden Vorschriften, die der Implementierung von Gesetzen dienen, wird vom Staatsrat und seinen entsprechenden Abteilungen vorgenommen. Rechtsmaterien, die per Gesetz geregelt werden müssen, für die aber noch kein Gesetz erlassen wurde, kann der Staatsrat durch Verwaltungsrechtsnormen festlegen, wenn er dazu vom NVK oder seinem Ständigen Ausschuss ermächtigt wird. ${ }^{31}$ In Art. 89 Abs. 1 der Verfassung heißt es außerdem, dass der Staatsrat befugt ist, gemäß der Verfassung und den Gesetzen Verwaltungsmaßnahmen und Verwaltungsrechtsnormen festzusetzen. Diese Formulierung ist sehr vage und lässt einen großen Interpretationsspielraum zu.

In juristischen Kreisen der V.R. China ist die Frage strittig, ob es sich hier um eine allgemeine Ermächtigung zur Festlegung von Exekutivnormen handelt oder ob der Staatsrat eine ausdrückliche gesetzliche Ermächtigung benötigt. ${ }^{32}$ Damit verbunden ist die Frage, ob der Staatsrat eine eigene Rechtsetzungsbefugnis besitzt oder nicht.

Der Rechtswissenschaftler Cai Dingjian vertritt die Ansicht, dass nur der NVK und sein Ständiger Ausschuss Gesetzgebungsbefugnisse besitzen, weil nur ein Verfassungsorgan diese Kompetenz haben kann. Schließlich stelle der NVK im Vergleich zu Staaten mit Gewaltenteilung die Legislative dar. Zwar könnten der Staatsrat und die Volkskongresse Rechtsnormen verabschieden, dies sei aber nur aufgrund einer Ermächtigung durch den NVK möglich. ${ }^{33}$

31 Art. 56 ff. des Gesetzgebungsgesetzes. Vgl. Gu, Angran, Einführung zum Gesetz der V.R. China über die Gesetzgebung (Zhongguo Renmin Gongheguo lifa fa jinhua), Rechtsverlag, Beijing, 2000, S. 14.

Gu, Angran (Fn. 31), S. 17; Li, Buyun, Several Issues Related to the Drafting of The Legislative Law of the People's Republic of China, in: Social Science in China, Heft Herbst 1998, S. 144 ff.; Qiao, Xiaoyang (Fn. 23), S. $203 \mathrm{ff}$. petenz (Lifaquan gailun), in: Forum für Politik und Recht (Zhengfa luntan), Beijing, Heft 6, S. 10 ff., S. 15. 
Nach der überwiegend vertretenen Meinung ${ }^{34}$ besitzt der Staatsrat eine delegierte Gesetzgebungsbefugnis. Auch wenn viele Verwaltungsrechtsnormen auf Grundlage einer Ermächtigung des NVK erlassen seien und der Grundsatz des Gesetzesvorrangs (falü youxian) im Gesetzgebungsgesetz verankert sei, ${ }^{35}$ könne die Exekutive ohne Gesetzesgrundlage Verwaltungsrechtsnormen verabschieden. ${ }^{36}$ In der Praxis übt die Regierung jedenfalls ein selbständiges Rechtsetzungsrecht aus. Hierin unterscheiden sich die chinesischen Verwaltungsrechtsnormen gravierend von den deutschen Rechtsverordnungen, die nur auf Grund einer gesetzlichen Ermächtigung von der Exekutive verabschiedet werden können. ${ }^{37}$

Ein weiteres Problem betrifft die Weitergabe einer Ermächtigung. Verwaltungsorgane geben die Ermächtigung in Teilen weiter. Auf diese Weise werden sogar nicht staatliche Organisationen zur Rechtsetzung ermächtigt. ${ }^{38}$ Zwar ist in $\S 10$ des Gesetzgebungsgesetzes festgelegt, dass Zweck und Umfang einer Ermächtigung eindeutig zu sein haben, aber von chinesischen Rechtswissenschaftlern ${ }^{39}$ wird eine weitere rechtliche Konkretisierung bezüglich des Adressatenkreises, des Inhalts und der Form von Ermächtigungen gefordert.

In der Normenhierarchie stehen die Verwaltungsrechtsnormen hinter den Gesetzen und haben Vorrang vor den von Volkskongressen und -regierungen der verschiedenen Ebenen $^{40}$ gesetzten Normen. ${ }^{41}$

Gesetze und Verwaltungsrechtsnormen sind im "Amtsblatt des Staatsrates" (guowuyuan gongbao $)^{42}$ und in Form von Jahresbänden in der amtlichen "Sammlung von Gesetzen und

Li, Buyun (Fn. 32), S. 145; Lin, Feng (2000), Constitutional Law in China, Hongkong, S. 80; Zhang, Zhongqiu/Zhang, Mingxin (Fn. 22), S. 5.

Art. 79 des Gesetzgebungsgesetzes.

Gu, Angran (Fn. 31), S. 14; Lin, Feng (Fn. 34), S. 79.

37 Art. 80 Abs. 1 GG.

Hu, Jianmao, Diskurs über das Verfassungsrecht, Verlag der Chinesischen Universität für Politik und Recht, Beijing, 1999, S. 240.

39

Hu, Jianmao (Fn. 38), S. 244 f.

40

Der Verwaltungsaufbau in der V.R. China ist in fünf Stufen geteilt: 1. Zentralregierung, 2. Provinzen, autonome Regionen und regierungsunmittelbare Städte (PAS), 3. Bezirke, 4. Kreisebene und 5. Gemeindeebene; vgl. Ebsen, Ingwer, Zur Entstehung eines Verwaltungsrechts in der Volksrepublik China, in: Verfassung und Recht in Übersee (VRÜ) 1999, S. 434 ff., 438.

42 Qiao, Xiaoyang (Fn. 23), S. 203 ff.

Das Amtsblatt wird vom Geschäftsbüro des Staatsrates (guowuyuan bangongting) herausgegeben und in der Regel mit GGB abgekürzt. 
Bestimmungen der V.R. China" (Zhongguo Renmin Gongheguo fagui huibian) ${ }^{43}$ zu veröffentlichen. 44

Abbildung 1: Normenhierarchie

\begin{tabular}{|l|l|}
\hline Folgende Instanzen erlassen & folgende Normen (in Rangordnung) \\
\hline Nationaler Volkskongress & Verfassung und grundlegende Gesetze \\
\hline Ständiger Ausschuss des NVK & Gesetze, die nicht grundlegend sind \\
\hline Staatsrat & Verwaltungsrechtsnormen (xingzheng fagui) \\
\hline Volkskongresse der PAS & Territoriale Rechtsnormen (difang fagui) \\
\hline $\begin{array}{l}\text { Territoriale Volksregierungen } \\
\text { der verschiedenen Ebenen }\end{array}$ & $\begin{array}{l}\text { Vorschriften (guizhang) } \\
\text { interne }=\text { Satzung oder externe } \\
(\text { (weibu })\end{array}$ \\
\hline
\end{tabular}

(Eigene Darstellung)

\subsection{Rechtsetzungsverfahren territorialer Rechtsnormen}

Territoriale Rechtsnormen (difangxing fagui) werden von Volkskongressen auf der Ebene der Provinzen, Autonomen Regionen und regierungsunmittelbaren Städten (im Folgenden PAS) sowie von Volkskongressen relativ großer Städte festgelegt. ${ }^{45}$ Als relativ groß werden Städte bezeichnet, die Sitz der Volksregierung einer Provinz, Autonomen Region oder Hauptsitz einer Sonderwirtschaftszone ${ }^{46}$ sind, oder wenn sie vom Staatsrat als relativ große Stadt ermächtigt wurden, territoriale Rechtsnormen festzulegen. ${ }^{47}$ Wenn diese Städte territoriale Rechtsnormen erlassen, müssen sie von dem jeweiligen Volkskongress der PASEbene genehmigt werden. ${ }^{48}$

Die Sammlung wird vom Rechtsamt des Staatsrates herausgegeben und in der Regel mit FGHB abgekürzt. Sie erscheint jährlich im Verlag des chinesischen Rechtssystems (Zhongguo fazhi chubanshe), Beijing.

Vgl. Art. 62 des Gesetzgebungsgesetzes.

Art. 99, 100 der Verfassung; Art. 63 des Gesetzgebungsgesetzes.

46

Seit Anfang der 80er Jahre wurden Sonderwirtschaftszonen geschaffen, die als Exportzonen dienen, um ausländisches Kapital anzuziehen.

47

Qiao, Xiaoyang (Fn. 23), S. 230 f.

Art. 63 des Gesetzgebungsgesetzes. 
Die territorialen Rechtsnormen dürfen nicht gegen die Verfassung, Gesetze oder Verwaltungsrechtsnormen verstoßen. Abgesehen von Gegenständen, die gemäß $§ 8$ des Gesetzgebungsgesetzes durch Gesetz geregelt werden müssen, dürfen lokale Volksregierungen jene Gegenstände festlegen, die noch nicht durch ein Gesetz oder eine Verwaltungsrechtsnorm geklärt sind. Außerdem können sie territoriale Rechtsnormen verabschieden, die für die Durchführung von Gesetzen oder für die lokale Regierungsarbeit benötigt werden. ${ }^{49}$ Territoriale Rechtsnormen sind gemäß $§ 70$ des Gesetzgebungsgesetzes in den entsprechenden Amtsblättern der Ständigen Ausschüsse zu veröffentlichen. ${ }^{50}$

Die verschiedenen Abteilungen des Staatsrats, zu denen auch die einzelnen Ministerien gehören, und die Volksregierungen der PAS sowie der relativ großen Städte inklusive der dazugehörigen Abteilungen können aufgrund einer Ermächtigung durch Gesetz oder Verwaltungsrechtsnorm Vorschriften (guizhang) erlassen. ${ }^{51}$ Diese Vorschriften lassen sich in interne und externe unterteilen. Interne Vorschriften entsprechen den deutschen Satzungen, während die Externen Verwaltungsvorschriften sind. ${ }^{52}$ Verwaltungsvorschriften sollen Gegenstände regeln, bei denen die Voraussetzungen für den Erlass von Verwaltungsrechtsnormen noch nicht gegeben sind. ${ }^{53}$ Dass heißt, sie sollen gemäß den Gesetzen und Verwaltungsrechtsnormen, die noch kein konkreten Regelungen vorsehen, beispielsweise wirtschaftliche Situationen, die sich in absehbarer Zeit weiter entwickeln werden, ordnen. In diesen Fällen reguliert die Verwaltungsvorschrift eine Übergangsphase. ${ }^{54}$

Die territorialen Rechtsnormen, die von den Volkskongressen der PAS erlassen werden, haben Vorrang vor den Vorschriften, die von der lokalen Exekutive und deren Behörden festgelegt werden. ${ }^{55}$ Der Staatsrat kann von Ministerien, Kommissionen und Verwaltungsbehörden gesetztes Recht aufheben. ${ }^{56}$ In der Praxis widersprechen sowohl territoriale Rechtsnormen als auch Vorschriften den Gesetzen. ${ }^{57}$

Art. 63, 64 des Gesetzgebungsgesetzes.

Vgl. beispielsweise die Internetseite des Amtsblatts des Ständigen Ausschusses in Shanghai: http://www.spcsc.sh.cn/rdgb.html

Art. 90 Abs. 2 der Verfassung; Art. 71 des Gesetzgebungsgesetzes. Qiao, Xiaoyang (Fn. 23), S. 265.

Art. 8 der "Regeln über das Festlegungsverfahren für Vorschriften" (guizhang zhiding chengxu tiaoli) vom 16.11.2002, GGB 2002, Heft 1, S. 7 ff. xiaoli), in: Forum für Politik und Recht (Zhengfa luntan) 1999, Beijing, Heft 6, S. 75 ff., 77; Ying, Songnian (Fn. 25), S. 9; Tanner, Murray Scott (Fn. 9), S. 129. 
Die Vorschriften müssen gemäß $§ 77$ des Gesetzgebungsgesetzes im Amtsblatt des Staatsrates bzw. der entsprechenden Abteilungen oder der territorialen Volksregierung veröffentlicht werden.

\section{Terminologie der einzelnen Normen}

Gesetze werden fast immer auch als Gesetze (falü) bezeichnet. Zu den wenigen Ausnahmen zählen die "Allgemeinen Grundsätze des Zivilrechts der V.R. China"58 vom 12.4.1986. Die Terminologie der anderen Rechtsnormen sollte mit den "Vorläufigen Regeln über das Festlegungsverfahren von Verwaltungsrechtsnormen”, die am 21.4.1987 vom Staatsrat erlassen wurden, vereinheitlicht werden. Diese wurden nach Verabschiedung des Gesetzgebungsgesetzes durch die "Regeln über das Festlegungsverfahren von Verwaltungsrechtsnormen" " vom 16.11.2001 ersetzt. ${ }^{61}$ Danach sind Verwaltungsrechtsnormen hauptsächlich "Regeln" (tiaoli), "Bestimmungen" (guiding) und "Maßnahmen" (banfa). ${ }^{62}$ Dabei sind "Regeln" (tiaoli) in der Normenhierarchie höher eingestuft als die übrigen Normen, weil sie nur vom Staatsrat und nicht von Ministerien, Kommissionen oder lokalen Volksregierungen verabschiedet werden dürfen. ${ }^{63}$ Wenn der Staatsrat aufgrund einer Ermächtigung des NVK Verwaltungsrechtsnormen erlässt, sollen diese als "Vorläufige Regeln" (zanxing tiaoli) oder "Vorläufige Bestimmungen" (zanxing guiding) bezeichnet werden, um den Rangunterschied zu kennzeichnen.

In den "Regeln über das Festlegungsverfahren für Vorschriften"65 werden keine Ausführungen über die Terminologie der lokalen Vorschriften vorgenommen. Generell werden auf dieser Ebene ebenfalls Bestimmungen (guiding) sowie Maßnahmen (banfa) verabschiedet,

Zhonghua Renmin gonghehuo minfa tongze, GGB 1986, S. 371ff.; engl. Übersetzung in: Laws of the People's Republic of China 1983-1986, Beijing, S. 225 ff.; dt. Übersetzung von Münzel, Frank in: Chinas Recht (Übersetzungen chinesischer Gesetzestexte, hrsg. von Frank Münzel, im folgenden: CR) 12.4.1986/1.

Xingzheng fagui zhiding chengxu zanxing tiaoli, GGB 1987, S. 454 ff. Teile sind in dt. Übersetzung von Heuser, Robert, Die Öffentliche Verwaltung (DÖV) 1988, S. 24.

60

Xingzheng fagui zhiding chengxu tiaoli, GGB 2002, Heft 1, S. 5 ff.

61

Art. 37 der "Regeln über das Festlegungsverfahren von Verwaltungsrechtsnormen".

Vgl. Zou, Yu/Gu, Ming, Großes Lexikon der Rechtswissenschaft (Faxue da cidian), Verlag der Universität für Politik und Recht, Beijing, 1992, S. 567.

Art. 4 der "Regeln über das Festlegungsverfahren von Verwaltungsrechtsnormen".

$Y e$, Bifeng, Verwaltungsrechtswissenschaft (Xingzheng faxue), Verlag der Universität Wuhan, Wuhan, 1996, S. 142 f.

65

Guizhang zhiding chengxu tiaoli vom 16.11.2002, GGB 2002, Heft 1, S. 7 ff. 
aber es dürfen keine "Regeln" (tiaoli) erlassen werden. ${ }^{66}$ Eine Unterscheidung von Verwaltungsrechtsnormen und Vorschriften ist nur deshalb möglich, weil auf der offiziellen Version der Rechtsnorm das verabschiedende Organ zu verzeichnen ist. ${ }^{67}$

\section{Auslegung}

Der Ständige Ausschuss des NVK ist gemäß Art. 67 Abs. 4 der Verfassung für die Auslegung $_{70}^{68}$ von Gesetzen verantwortlich. ${ }^{69}$ Diese Auslegungen haben gesetzesgleiche Wirkung. ${ }^{70}$

Das Höchste Volksgericht hat gemäß $§ 33$ des "Gesetzes der V.R. China über die Organisation der Volksgerichte" ${ }^{\text {, }}$, das am 1.7.1979 verkündet und am 2.9.1983 revidiert wurde, die Befugnis, Gesetze und Beschlüsse in Gerichtsverfahren zu interpretieren. Ebenso hat die Oberste Volksstaatsanwaltschaft gemäß "Beschluss des Ständigen Ausschusses des NVK über die Stärkung der Arbeit der Gesetzesinterpretation" "72 vom 10.6.1981 die Kompetenz, im Rahmen ihrer Tätigkeit Fragen zur Anwendung von Gesetzen zu beantworten. Wenn Auslegungen des Höchsten Volksgerichts und der Volksstaatsanwaltschaft miteinander kollidieren, entscheidet der Ständige Ausschuss des NVK darüber. Die Interpretationen der Gerichte und der Volksstaatsanwaltschaft sind von großer praktischer Bedeutung. ${ }^{73}$

Allerdings beziehen sich diese Auslegungen häufig nicht nur auf Gerichtsfälle, sondern es werden Gesetze generell interpretiert oder neue Prinzipien und Standards für die Rechtsprechung anhand von konkreten Fällen eingeführt. Für diese Gesetzesauslegungen der

Art. 6 der "Regeln über das Festlegungsverfahren für Vorschriften" (guizhang zhiding chengxu tiaoli) vom 16.11.2002, GGB 2002, Heft 1, S. 7 ff.

Art. 30 der "Regeln über das Festlegungsverfahren für Vorschriften" (guizhang zhiding chengxu tiaoli) vom 16.11.2002, GGB 2002, Heft 1, S. 7 ff.

Vgl. von Senger, Harro, Einführung in das chinesische Recht, München, 1994, S. 175 ff.; Zhang Zhiming, The Chinese System of Interpreting the Law, in: Social Science in China, Heft Herbst 1998, Beijing, S. $131 \mathrm{ff}$.

Art. 42 ff. des Gesetzgebungsgesetzes; vgl. Cai, Dingjian, Functions of the People's Congress in the process of implementation of law, in: Chen, Jianfu/Li, Yuwen/Otto, Jan Michiel, Implementation of Law in the People's Republic of China, 2002, S. $35 \mathrm{ff}$., $36 \mathrm{ff}$. Art. 47 des Gesetzgebungsgesetzes.

Quangguo Renmin Daibiao Dahui Changwu Weiyuanhui guanyu jiaqiang falü jieshi gongzuo de jueding, FGHB 1981, S. 27 f.

73 Cai, Dingjian (Fn. 69), S. 38. 
Gerichte existiert keine rechtliche Grundlage, und sie sind daher umstritten. ${ }^{74}$ In der Praxis besitzen sich dennoch eine große Bedeutung, da kein Gericht von diesen Interpretationen abweichen würde.

\section{Politische Richtlinien ${ }^{75}$}

Der Gesetzgebungsprozess unterliegt in der V.R. China der Kontrolle durch die Kommunistische Partei. Bereits im 3. Paragraphen des Gesetzgebungsgesetzes heißt es, dass die Gesetzgebung unter der Führung der Kommunistischen Partei zu geschehen hat.

Zunächst wird dies auf personelle Weise sichergestellt. Der seit März 2003 amtierende Präsident des NVK, Wu Bangguo, ${ }^{76}$ gehört zum Ständigen Ausschuss des Politbüros. Außerdem sind drei weitere Vizepräsidenten des NVK Mitglieder des Politbüros. Darüber hinaus sind knapp zwei Drittel der Delegierten des NVK Mitglieder in der Kommunistischen Partei. ${ }^{77}$ Viele der Delegierten zählen zu den Kadern (ganbu). ${ }^{78}$

Die Kommunistische Partei erlässt Politnormen in Form von "Mitteilungen" (tongzhi) oder "Richtlinien" (zhengce). ${ }^{79} \mathrm{Zu}$ diesen Richtlinien der Kommunistischen Partei gehörten früher die wichtigsten Rechtsnormen der V.R. China. Allerdings haben sie keine rechtliche Bindungskraft und beschränken sich in den letzten Jahren meistens auf allgemeine Grundsätze, die dann aber eine erhebliche Rolle spielen, wie z.B. der "Beschluss der Zentrale über einige Fragen zur Errichtung einer sozialistischen Marktwirtschaft" 80 vom 14.11.1993. ${ }^{81}$ Meistens handelt es sich um Parteibeschlüsse auf Parteitagen, die eine nationale Politik bestimmen. Parteibeschlüsse auf Provinzebene sind dagegen selten.

Zhang, Zhiming, (Fn. 68), S. 131 ff., S. 140; Chen, Jianfu, Chinese Law - Towards an Understanding of Chinese Law, Its Nature and Development, Den Haag, 1999, S. 108 f. Vgl. von Senger, Harro (Fn. 68), S. 203 ff.

Zur Person siehe: China aktuell, November 2002, Hamburg, S. 1280.

Für den 10. NVK, CD vom 28.2.2003; vgl. für den 9. NVK Cai, Dingjian (Fn. 8), S. 227.

Ding, Shusu/Zhang, Ying, Diskussion über die Aufsichtsmacht der Nationalen Volkskongresse und weiterer Verbesserungen in unserem Land (Shilun woguo renda jianduquan jiqi wanshan), in: Forum für Politik und Recht (Zhengfa luntan) 1998, Beijing, Heft 6, 48 ff., 52.

Shi, Tanjing, Untersuchung zum Recht der Sozialen Sicherheit (Shehui baozhang fa yanjiu), Verlag für Recht, Beijing, 2000, S. 54.

Zhonggong Zhongyang guanyu jianli shehui zhuyi shichang jingji tixi ruogan wenti de jueding, RMRB vom 17.11.1993.

Vgl. von Senger, Harro (Fn. 68), S. 198ff.; Tanner, Murray Scott (Fn. 9), S. 51 ff. 


\section{Normenkontrolle}

Die formelle Rangordnung der Rechtsquellen und der Vorbehalt des Gesetzes implizieren das Prinzip des Vorrangs der ranghöheren Rechtsnorm. Eine Normenkontrolle im westlichen Sinne existiert in der V.R. China nicht. Zumal keine Institutionen, wie beispielsweise Verfassungsgerichte, etabliert wurden, die eine unabhängige Prüfung über mögliche Kollisionen von Rechtsnormen mit der Verfassung vornehmen könnten. Indes existiert ein System abgestufter Aufhebungsrechte der jeweils übergeordneten Staatsorgane.

Der Nationale Volkskongress hat gemäß Art. 62 Abs. 11 der Verfassung die Befugnis, Gesetze und Entscheidungen des Ständigen Ausschusses aufzuheben. Dem Ständigen Ausschuss obliegt es gemäß Art. 67 Abs. 7 der Verfassung, Verwaltungsrechtsnormen des Staatsrats, die im Widerspruch zur Verfassung oder anderen Gesetzen stehen, aufzuheben. Außerdem kann er territoriale Rechtsnormen, die mit der Verfassung, Gesetzen oder Verwaltungsrechtsnormen kollidieren, annullieren. ${ }^{82}$

Für die Annullierung von Vorschriften (guizhang) der Ministerien und Kommissionen des Staatsrats ist der Staatsrat zuständig. Außerdem kann er Verwaltungsrechtsnormen und Vorschriften der lokalen Volkskongresse und Regierungsorgane aufheben. ${ }^{83}$

Die Ständigen Ausschüsse der lokalen Volkskongresse von der Kreisebene aufwärts heben territoriale Rechtsnormen der gleichen Ebene und Vorschriften der Volkskongresse der nächstniedrigeren Ebene auf. ${ }^{84}$ Eine nationale Regelung über die abgestuften Aufhebungsrechte unter der Bezirksebene existiert nicht.

\section{Aufsichtsfunktionen des NVK}

Die chinesische Verfassung legt keine Gewaltenteilung fest. Zwar werden dem NVK, dem Staatsrat und dem Obersten Volksgericht jeweils die Aufgaben und Funktionen der Legislative, Exekutive und Judikative zugewiesen. Trotzdem werden diese drei Gewalten nicht klar voneinander getrennt. Darin wird eine konkrete Realisierung der politischen Idee gesehen wird, dass das Volk allein den Staat führen soll. ${ }^{85}$ Aus diesem Grund fungiert der Ständige Ausschuss des NVK auch als Aufsichtsorgan, der gemäß Art. 67 der Verfassung die Durchführung der Verfassung überprüft sowie die Arbeit des Staatsrats, der Zentralen

82

83

84

85

Art. 64 Abs. 8 der Verfassung.

Art. 89 Abs. 13 und 14 der Verfassung.

Art. 104 der Verfassung.

Tomson, Edgar/Su, Jyun-hsyong (1972), Regierung und Verwaltung der Volksrepublik China, Köln, S. 118. 
Militärkommission, des Obersten Volksgerichts und der Obersten Volksstaatsanwaltschaft überwacht.

\subsection{Aufsicht über die Gesetzesdurchführung}

Eine weitere Aufgabe des Ständigen Ausschusses des NVK ist die Überwachung der Gesetzesimplementierung. In der Praxis haben sich Inspektionsreisen als Überwachungstätigkeit des Ständigen Ausschusses des NVK herausgebildet. Die historischen Wurzeln dieser Inspektionen liegen in den gegenseitigen Besuchen von Vertretern der Volkskongresse. Ab Mitte der 80iger Jahre wurden diese Besuchsreisen dann zu Inspektionsreisen umfunktioniert. Die Überwachungsaktivitäten sind im Prinzip nicht geregelt, sondern folgen einem Gewohnheitsrecht, das in der Praxis entstand und später legalisiert wurde. ${ }^{86}$

Im Jahr 1993 wurden "Einige Bestimmungen zur Verstärkung der Überwachung und Kontrolle über die Durchführung von Gesetzen des Ständigen Ausschusses des NVK”, ${ }^{, 87}$ erlassen, welche die Überwachung standardisieren und Lösungsmöglichkeiten von Konflikten vorsehen. Die Inspektionsreisen und die zu inspizierenden Gesetze werden ein Jahr im voraus vom Ständigen Ausschuss des NVK und seinen speziellen Ausschüssen festgelegt. Die zu untersuchenden Behörden werden nach Bekanntgabe des Überprüfungsplans informiert. Die Gruppe der Inspekteure wird aus den genannten Ausschüssen rekrutiert und umfasst normalerweise nicht mehr als sieben Personen. ${ }^{88}$

Die Inspektionen dienen der Überprüfung der Rechtsumsetzung und erst in zweiter Linie der Beseitigung von Rechtskollisionen. Dieser Fokus scheint gerechtfertigt, da teilweise nur 10 bis $20 \%$ der Gesetze überhaupt angewendet werden. ${ }^{89}$ Schwere Rechtsverstöße sind der Vorsitzendenkonferenz des Ständigen Ausschusses des NVK zu melden. Diese beauftragen wiederum eine Gruppe, die aus Mitgliedern der verschiedenen Ausschüsse des NVK besteht, in der Angelegenheit zu ermitteln. In der Praxis versuchen diese Inspektoren Kompromisse mit den entsprechenden lokalen Behörden zu erzielen, da sie offene Kritik oder direkte Maßnahmen meiden wollen. Diese setzen den entsprechenden Behörden und Abteilungen Fristen zur Lösung des Problems. Konkrete Rechtsfolgen sind in den oben

Cai, Dingjian (Fn. 69), S. 42.

Quanguo Renmin Daibiao Dahui Changweiyuanhui guanyu jiaqiang dui falü shishi qingkuang jiancha jiandu de ruogan guiding - am 2.9.1993 vom Ständigen Ausschuss des NVK verabschiedet; FGHB 1993, S. 300 ff.

Punkt 3 ff. "Einige Bestimmungen zur Verstärkung der Überwachung und Kontrolle über die Durchführung von Gesetzen des Ständigen Ausschusses des NVK" (Fn. 86).

Zhang, Zhongqiu/Zhang, Mingxin (Fn. 22), S. 5. 
genannten Bestimmungen nicht vorgesehen. Daher ist die Effizienz dieser Inspektionen fraglich. ${ }^{90}$

\section{2. $\quad$ Überwachung der Tätigkeit von Rechtspflegeorganen}

Eine weitere Kontrollaufgabe des NVK ist die Überwachung der Arbeit von Volksgerichten und -anwaltschaften. ${ }^{91}$ Auch an diesem Punkt zeigt sich, dass keine Gewaltenteilung in der V.R. China herrscht. Konkrete Richtlinien, wie die Überwachung von Urteilen etc. auszusehen hat, existieren nicht. Jedoch nimmt die praktische Bedeutung der Überwachung individueller Fälle zu, da Gerichtsurteile im Rahmen der juristischen Reformen immer wichtiger werden und damit einhergehend Korruption und Missbrauch der Justizmacht zu einem gravierenden gesellschaftlichen Problem geworden sind. Da es aber keine einheitliche, rechtliche Regelung über die Überprüfung individueller Rechtsfälle gibt, praktizieren die einzelnen Volkskongresse diese Kontrollfunktion in verschiedenartiger Weise und mit unterschiedlichen Ergebnissen. Aus diesem Grund wurde im Mai 1999 eine Kommission zur Erarbeitung von Bestimmungen bei schwerwiegenden Rechtsverstößen von Volksgerichten und -anwaltschaft gegründet. ${ }^{92}$

Im Rahmen der Diskussion über diese Bestimmungen zeichneten sich äußerst konträre Meinungen $a b$, bei der Einige eine strengere Kontrolle der Justizorgane befürworteten, während andere diese strikt ablehnten. ${ }^{93}$ Die Gegner ${ }^{94}$ einer Verstärkung der justiziellen Kontrolle durch den NVK argumentieren, dass die Unabhängigkeit der Justiz dadurch untergraben würde. Außerdem würde die Überwachung durch den NVK bedeuten, dass Aufgaben der Legislative und der Judikative durch ein Organ ausgeübt würden. Damit würde der NVK die verfahrensrechtlichen Regelungen treffen, die er dann selber zu befolgen hätte. Dies würde einer internen Prüfung gleichen. ${ }^{95}$ Ferner seien die Mitglieder des NVK juristisch nicht genügend geschult, eine Überwachung von Mitgliedern des NVK bedeute die Überwachung durch Individuen, dadurch würde die Korruption gefördert, da

94 Fei, Shancheng, Diskussion über die Wahl des Systems zur Überprüfung von verfassungswidrigen Handlungen in unserem Land (Shilun wo weixian shencha zhidu de moshi xuanze), in: Forum für Politik und Recht (Zhengfa luntan) 1999, Beijing, Heft 2, S. 2 ff., 5; vgl. Cai, Dingjian (Fn. 69), S. 51 . 
viele NVK-Mitglieder Inhaber von Unternehmen seien und schon jetzt in zahllose Rechtsfälle involviert wären. ${ }^{96}$

Im Gegensatz dazu argumentieren die Befürworter ${ }^{97}$, welche die Kontrollbefugnisse des NVK stärken wollen, dass gerade die Korruption durch stärkere Kontrolle unterbunden werden könnte. Dass es den Delegierten an juristischen Kenntnissen mangele, sei eine Ausrede, da sie entsprechend geschult werden könnten. Der Widerspruch gegen eine Stärkung des NVK wurde als derart überzeugend erachtet, dass die oben genannte Bestimmung bis heute nicht verabschiedet wurde. 98 Auch die Verabschiedung eines "Kontrollgesetzes" (jiandufa) ist vorgesehen, aber noch nicht erfolgt. ${ }^{99}$ Die Lösung des Problems steht bisher noch aus.

\subsection{Aufbau eines Verfassungsgerichts?}

Um die Aufgaben von Legislative und Judikative bei der Normenkontrolle und der Aufsicht über die Rechtsprechung zu trennen, könnte ein Verfassungsgericht eingerichtet werden. Allerdings wurde schon bei den Arbeiten am Entwurf zum Gesetzgebungsgesetz die Möglichkeit, ein "Verfassungsgericht" (xianfa fayuan) oder einer "Verfassungskommission" (xianfa weiyuanhui) unter der Leitung des NVK zu errichten, abgelehnt. Argumentiert wurde, dass politische Strukturreformen im Gesetzgebungsgesetz nicht vorgesehen sind. ${ }^{100}$

Im Zusammenhang mit dem Aufbau eines Rechtsstaats ${ }^{101}$ und der Korruptionsbekämpfung ist es notwendig geworden, Individuen einen größeren Rechtsschutz zu gewähren und eine effektive Normenkontrolle durchzuführen. Aus diesen Gründen wird von chinesischen Rechtswissenschaftlern ${ }^{102}$ die Errichtung eines Verfassungsgerichts in der Fachliteratur weiter diskutiert werden. Die Mehrheit der Juristen ist der Ansicht, ${ }^{103}$ dass eine Verfassungskommission oder ein Verfassungsgericht unter der Führung des NVK zu errichten sei.

100 Vgl. Heuser, Robert (Fn. 27), S. 63 f.; Li, Buyun (Fn. 32), S. 153 f.

101 In Art. 5 der Verfassung bekennt sich die V.R. China seit 1999 dazu, einen "sozialistischen Rechtsstaat" (shehui zhuyi fazhi guojia) aufzubauen.

102 Zhang, Zhongqiu/Zhang, Mingxin (Fn. 22), S. 9.

103 Fei, Shancheng (Fn. 94), S. 11; Li, Buyun (Fn. 32), S. 153; Zhang, Zhongqiu/Zhang, Mingxin (Fn. 22), S. 9. 
Eine Minorität ist der Meinung ${ }^{104}$, die Verfassungskontrolle sei Aufgabe der Politischen Konsultativkonferenz $^{105}$. Historisch betrachtet, hätte die Politische Konsultativkonferenz den NVK im Jahr 1954 erst geschaffen, so dass die Konsultativkonferenz prädestiniert wäre, die Normenkontrollen bezüglich der Verfassung auszuüben. ${ }^{106}$ Durch die Konsultativkonferenz könne eine demokratische Kontrolle stattfinden, die auch wirtschaftlichen Interessen Einhalt gebiete.

Die Aufwertung der Politischen Konsultativkonferenz als Gegengewicht zur Kommunistischen Partei ist im Rahmen der Bekämpfung von Korruption äußerst aktuell. Die Konstruktion, die Politische Konsultativkonferenz als Organ zur Normenkontrolle zu etablieren, ist einzigartig und könnte für die V.R. China ein gangbarer Lösungsweg sein.

\section{Schlussbetrachtung}

Seit einigen Jahren ist das Bemühen um eine stärkere Rechtsvereinheitlichung zu erkennen, das jedoch durch eigenmächtige Rechtsetzung lokaler Regierungen konterkariert wird; denn der in der V.R. China herrschende "Lokalprotektionismus" (difang zhuyi) verleitet manchen lokalen Potentaten dazu, seine rechtlichen Kompetenzen zu überschreiten und Normen zu erlassen, die gegen geltende Gesetze verstoßen. ${ }^{107}$ Aber auch der in China existierende "Behördenprotektionismus" (bumen zhuyi) führt zu Rechtsverletzungen und Streitigkeiten über die Rechtsetzungskompetenzen. ${ }^{108}$ Die Verabschiedung des Gesetzgebungsgesetzes soll diesen Missstand beheben, es ist aber gerade in Bezug auf die Festlegung territorialer Normen vage formuliert.

Bei den Ausarbeitungen zum Gesetzgebungsgesetz konnten sich anfängliche Überlegungen, ein Verfassungsgericht ${ }^{109}$ aufzubauen, nicht durchsetzen. Wenn tatsächlich ein unabhängiges Verfassungsgericht etabliert würde, müsste die chinesische Verfassung dahingehend geändert werden. Indes werden machtpolitische Gründe diesen Überlegungen entgegenste-

104 105 Zhu, Weijiu (Fn. 3), S. 145 ff.

Die Politische Konsultativkonferenz des Chinesischen Volkes (Zhongguo Renmin zhengzhi xieshang huiyi) wurde 1949 gegründet. Sie gehört weder zu einem Staatsorgan, noch ist sie eine allgemeine gesellschaftliche Organisation. Vielmehr ist sie eine Organisation der demokratischen Einheitsfront des chinesischen Volkes und damit Plattform der verschiedenen Parteien. Vgl. 106 Tomson, Edgar/Su, Jyun-hsyong (Fn. 85), 75 ff.

Zhu, Weijiu (Fn. 3), S. 145 f.; vgl. Chen, Jianfu (Fn. 74), S. 98 f.

107 Vgl. Gu, Angran (Fn. 31), 19 f., 78; von Senger, Harro (Fn. 68), S. 170 m. w. N.

108 Vgl. Li, Yuwen/Otto, Jan-Michiel (Fn. 2), S. 26.

109 Vgl. von Senger, Harro, Einführung in das chinesische Recht, München, 1994, S. 175 ff.; Zhang Zhiming (Fn. 68), S. $131 \mathrm{ff}$. 
hen. Es ist vielmehr zu erwarten, dass eine Verfassungskommission unter Leitung des NVK errichtet wird, die dann aber nicht unabhängig ist.

Die Verquickung legislativer und judikativer Aufgaben durch ein Organ, den NVK, ist dem westlichen Betrachter mit seinen Vorstellungen der Gewaltenteilung fremd. Im Gegensatz dazu wird die Machtkonzentration beim NVK in der V.R. China als Ausdruck der Herrschaft durch das Volk gesehen. Von einem westlichen Parlament unterscheidet sich der NVK aufgrund seiner vielfältigen Aufgaben und durch die nach wie vor bestehende Kontrolle der Kommunistischen Partei. Wie das Zitat des Legalisten Guanzi zeigt, dient die Festlegung des Rechtsetzungsverfahrens eher dazu, die Herrschaft der Kommunistischen Partei zu konsolidieren und weniger dem Bestreben, einen Rechtsstaat zu etablieren. 